\title{
A DETECTION-BASED FRAMEWORK FOR THE ANALYSIS OF RECYCLING IN TIRF MICROSCOPY
}

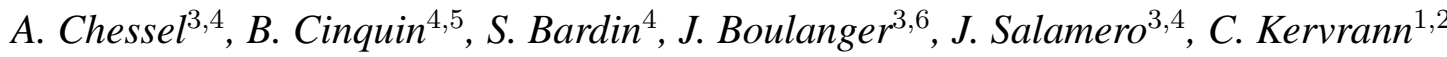

(1) INRIA Centre Rennes - Bretagne Atlantique, F-35042 Rennes

(2) INRA, UR341 Mathématiques et Informatique Appliquées, F-78352 Jouy-en-Josas

(3) "Cell and Tissue Imaging Facility" - IBISA, Institut Curie, F-75248 Paris

(4) UMR 144 CNRS - Institut Curie, F-75248 Paris

(5) Soleil Synchrotron, F-91192 Gif-sur-Yvette

(6) RICAM, A-4040 Linz, Austria

\begin{abstract}
Endocytosis/recycling and exocytosis are mechanisms conserved through evolution allowing cells to communicate with their external medium. In order to study these dynamic processes, the present work proposes a patch-based method for detecting recycling or exocytotic events at the Plasma membrane in fast TIRF microscopy combined with the computation of normalized temporal representations of those events. Evaluation, performed on TIRF sequences showing Transferrin receptor (TfR) recycling, validates a high detection rate fully compatible with an automatic data extraction and analysis of the plasma membrane recycling process.
\end{abstract}

Index Terms - patch-based, event detection, TIRFM, TfR, endocytosis/recycling

\section{INTRODUCTION}

Total internal reflection fluorescence microscopy (TIRFM) allows for the imaging of a very thin layer near the cell surface, at about 100 or $200 \mathrm{~nm}$ depth from a glass cell support. The dynamic study of events happening between the cell and the external medium, seen as sudden changes at the plasma membrane, is then possible. In particular, endocytosis/recycling pathways are among the mechanisms that allow communication of the cell with the external medium.

In this work, our focus is the systematic study of the recycling of fluorescent trans-membrane proteins. The recycling of an individual vesicle observed in TIRFM then typically corresponds to a sudden appearance as it docks to the plasma membrane. It is usually followed by a more or less slower decrease in fluorescence intensity as the targeted molecule vanishes in the medium, in the membrane plane or for peripheral molecules, when it dissociates from the vesicles.

Fast TIRF microscopy shows that as many as hundreds of such events may occur every minute at different locations within a single cell. Moreover, it is almost impossible at a glance to assess the degree of temporal and spatial homogeneity of these events. If one wishes to study multiple conditions or different molecular behaviors at the single cell level, yet overcoming the basic limitation of pure

Acknowledgments: The authors wish to thank the Nikon Imaging Centre@Institut Curie-CNRS, for providing us with up to date microscopy systems. A.C. is a recipient of a fellowship provided by the ANR BIOtechSan program, $\mathrm{MiCaD}$ project ((NANR-08-Biot-015-01) qualitative or manual approaches, automatic statistical analysis of heavy image series is required.

To this aim, the framework proposed here includes two steps. First a new patch-based change detection method is proposed to extract all the individual events from a TIRFM sequence; they are looked for as suddenly appearing bright spots by comparing pairs of images. Once detected, the recycling vesicle is supposed to be immobile and the second step is the computation, for each detected event, of a normalized temporal profile that will allow future statistical studies and comparison.

The paper is organised as followed. Section 2 presents the biological model and the experimental setup used to obtain the raw data. In Section 3, the detection method is described and quantitatively validated with respect to ground truths. Finally, Section 4 describes the proposed normalized representation applied to each detected temporal signal.

\section{BIOLOGICAL MODEL, EXPERIMENTAL SETUP AND IMAGE ACQUISITION}

While eukaryotic cells need to insure their integrity, they also have to communicate with their external medium. From yeast to human cells, mechanisms have been conserved through evolution that guarantee secretion, capture of diverse elements from the environment as well as recapture and shuttling of cellular receptors that allow selectivity of internalized molecules. Transferrin receptor is a hallmark of constitutively recycling transmembrane receptors $[1,2]$ whose expression and function is directly linked to cell division and accurate iron metabolism at the cellular level, through endocytosis and processing of extracellular iron charged transferrin [3]. As a live cell model to study this well known reporter molecule, chimeras have been engineered between TfR and fluorescent proteins for their introduction and expression in different cell lines.

To get deeper insights in the dynamics of proteins, such as TfR, recycling from intracellular membrane pools to the plasma membrane, relatively new optical techniques have been thoroughly used over the last decades. Total Internal Reflexion Fluorescence [4] microscopy based on the properties of evanescent waves propagation at the interface between two media with sufficiently different refractive indexes, is certainly the method of choice. This widely used approach demonstrated that transient fluorescence concentrations can occur close to or at the plasma membrane, suggesting that they may correspond to membrane sites specialized in endocytosis or exocytosis [5]. Among diverse molecular behaviors reported by previous 


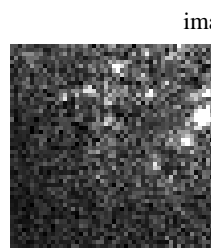

image pair
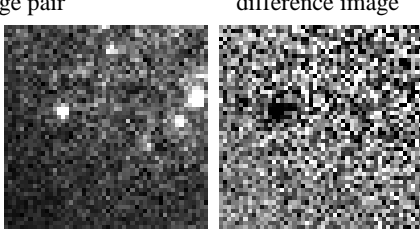

Fig. 1. Change detection in an image pair in TIRFM.

microscopy studies on this molecular model, plasma membrane concentration of fluorescently tagged proteins give rise to sudden spot appearance and/or disappearance.

By allowing the visualization of a single plane near the plasma membrane within a $100 \mathrm{~nm}$ narrow range of resolution in the $\mathrm{Z}$ axis, TIRFM permits to detect and decipher the progressive molecular steps corresponding to the docking of fluorescently labeled moving vesicles when they reach the plasma membrane, their fusion with this latter membrane and the biophysical property of diffusion, redistribution or endocytosis of the transmembrane proteins thus delivered at the cell surface.

For the experiments, M10 cells are transiently transfected with a plasmid encoding for the TfR fused to the PhLuorin [6]. Crossbow shaped micropattern constraining the cell are used; they allow for more stable experiment, from both biological and image analysis point of view. TIRFM sequences are acquired on a Nikon TE2000 inverted microscope equiped with a TIRF arm. We use a high aperture 100x TIRF objectif $(\mathrm{NA}=1.49)$. Image sequences were exclusively generated in stream mode of acquisiton, exposure time per frame is $100 \mathrm{~ms}$ for a 1 minute total duration of imaging (about 600 frames per series).

\section{MULTISCALE EVENT DETECTION}

In this section we propose an event detection framework from an image pair. This method will then be evaluated on the acquired TfR$\mathrm{pHl}$ sequences, each pair of images being tested successively.

\subsection{Theory and algorithm}

Consider a gray-scale image pair $u=(u(\mathbf{x}))_{\mathbf{x} \in \Omega}$ and $v=(v(\mathbf{x}))_{\mathbf{x} \in \Omega}$ defined over a bounded domain $\Omega \subset \mathbb{R}^{2}$. In order to test robustly the similarity between $u$ and $v$ at location $\mathbf{x}$, our idea is to predict a patch $\underline{u}(\mathbf{x})$ of size $n$ in $u$ from patches $\left(\underline{v}\left(\mathbf{x}_{i}\right)\right)_{\mathbf{x}_{i} \in B(\mathbf{x})}$ taken in the (fixed size) semi-local neighbourhood $B(\mathbf{x}) \subseteq \Omega$. In what follows, $N=|B(\mathbf{x})|$ will denote the number of elements observed at locations $\mathbf{x}_{i}$ 's in the second image $v$ and a vectorized image patch.$_{-}(\mathbf{x})$ is defined as a vector of pixel intensities ordered lexicographically in the square patch.

We propose a two-step approach to detect pixels with significant changes for a given patch size. The local decisions are furthermore analyzed in a multiscale framework by considering several patch sizes, mainly to detect unusual events in the whole image and to reduce the number of false alarms. More formally, let us consider a score $z\left(\mathbf{x}_{i}\right)=\left\|\underline{u}(\mathbf{x})-\underline{v}\left(\mathbf{x}_{i}\right)\right\|^{2}$ defined as the $L_{2}$ distance between the two patches $\underline{u}(\mathbf{x})$ and $\underline{v}\left(\mathbf{x}_{i}\right), \mathbf{x}_{i} \in B(\mathbf{x})$. The score is non-negative and the smaller the value of score is, the more the similar patches are. For a given patch size $n$, the two-step decision mechanism is as follows:

1. For each pixel $\mathbf{x}_{i} \in B(\mathbf{x})$, we compute a score $z\left(\mathbf{x}_{i}\right)$ and we make a binary decision $d\left(\mathbf{x}_{i}\right)$ with respect to a spatially-varying threshold $\tau(\mathbf{x}): d\left(\mathbf{x}_{i}\right)=1$ if $z\left(\mathbf{x}_{i}\right) \geq \tau(\mathbf{x})$ and 0 otherwise.
Let $N$ be the fixed size of neighbourhood and $L$ be the number of scales (patch sizes)

$\circ$ For $l=1 \cdots L$, compute

$$
\begin{aligned}
& \text { 1. } \tau_{l}(\mathbf{x})=\max \left(\sup _{\mathbf{y} \in B(\mathbf{x})}\left\|\underline{u}_{l}(\mathbf{x})-\underline{u}_{l}(\mathbf{y})\right\|^{2}, \tau_{0}\right) . \\
& \text { 2. } S_{N, l}(\mathbf{x})=\sum_{\mathbf{x}_{i} \in B(\mathbf{x})} \mathbb{1}_{\left\|\underline{u}_{l}(\mathbf{x})-\underline{u}_{l}\left(\mathbf{x}_{i}\right)\right\|^{2} \geq \tau(\mathbf{x}) .}
\end{aligned}
$$

○ Compute $\lambda_{N}=\frac{e^{-N}}{|\Omega|} \sum_{l=1}^{L} \sum_{\mathbf{y} \in \Omega} e^{S_{N, l}(\mathbf{y})}$

○ For $\mathbf{x} \in \Omega$, compute $\operatorname{PFA}(\mathbf{x})=1-\sum_{k=0}^{k_{D}(\mathbf{x})}\left(\lambda_{N}\right)^{k} \frac{e^{-\lambda_{N}}}{k !}$ and make the decision: $M C D(u, v)(\mathbf{x})=1$ if $\operatorname{PFA}(\mathbf{x}) \leq \varepsilon /|\Omega|$ and 0 otherwise.

Fig. 2. Change detection algorithm between images $u$ and $v$.

2. We make a collective decision from individual decisions $\left\{d\left(\mathbf{x}_{i}\right): \mathbf{x}_{i} \in B(\mathbf{x})\right\}$ at neighboring points [7, 8, 9, 10]. An intuitive choice is to count the total number of positive decisions made by individual pixels and denoted $S_{N}(\mathbf{x})=\sum_{\mathbf{x}_{i} \in B(\mathbf{x})} d\left(\mathbf{x}_{i}\right)$. Given $S_{N}(\mathbf{x})$, we declare that a change occurs at location $\mathbf{x}$ (hypothesis $\left.H_{1}\right)$ if all the scores are higher than $\tau(\mathbf{x})$ in the neighborhood $B(\mathbf{x})$ (maximum vote) and we select the hypothesis $H_{0}$ otherwise: $D(\mathbf{x})=1$ if $S_{N}(\mathbf{x})=N$ and $D(\mathbf{x})=0$ otherwise.

In this procedure, cooperation among neighbouring points will tend to enhance the ability to detect meaningful changes. Nevertheless, the detection results depend very much on the thresholds $\tau(\mathbf{x})$, the size $n$ of the patch and the size $N$ of the search window. In what follows, we give cues to interpret and set these parameters.

Estimation of spatially-varying thresholds $\tau(\mathbf{x})$ To derive adaptive thresholds for change detection, we define $\tau(\mathbf{x})$ as the highest score computed only from the reference image $u$ and neighborhoods $B(\mathbf{x})$ whose fixed size $N$ is related to the expected amplitude of background dynamics:

$$
\tau(\mathbf{x})=\max \left(\sup _{\mathbf{y} \in B(\mathbf{x})}\|\underline{u}(\mathbf{x})-\underline{u}(\mathbf{y})\|^{2}, \tau_{0}\right) .
$$

By introducing a minimal value $\tau_{0}$ defined as the average of the lowest scores computed over the image domain $\Omega$, we improve the robustness to low signal-to-noise ratios. In the proposed scene modeling, the background is assumed to be nearly static some irrelevant background dynamics described by stochastic processes. Let $\mathbf{s}(\mathbf{x}) \in \mathbb{R}^{2}$ be a zero-mean random shift (or displacement) vector. A patch $u(\mathbf{y})$ at random position $\mathbf{y} \in B(\mathbf{x})$ defined as $\mathbf{y}=\mathbf{x}+\mathbf{s}(\mathbf{x})$ will be potentially moved to the location $\mathbf{x}$ in the second image $v$ if no meaningful change occurs: $\underline{v}(\mathbf{x}) \equiv \underline{u}(\mathbf{y}-\mathbf{s}(\mathbf{x}))$. Hence, by defining $\tau(\mathbf{x})$ as in (1), variations due to motion in the background will not be considered as meaningful. These thresholds are computed directly from image data and the computation of the noise variance is not required.

The size $N$ of the search window depends on the motion amplitude of vesicles we do not want to detect. In the application, we set $N=3 \times 3$. This means we are testing dissimilarity in very local neighborhoods. Increasing the search window size should produce the same results since the missing or occluded patches are not visible in the second image for any location.

Multiscale decision fusion The multiscale approach is recommended traditionally to analyze several spatial contexts. In our case, a multiscale strategy enables to reduce the number of false alarms and to avoid the precise setting of the patch size $n$. Moreover, the decisions $D(\mathbf{x})$ are local and we need to consider the whole image 
for detecting unusual events when comparing the two images.

Let us consider $\mathcal{D}(\mathbf{x})=\left\{D_{1}(\mathbf{x}), \cdots, D_{L}(\mathbf{x})\right\}$ the set of binary decisions obtained at pixel $\mathbf{x}$, where $D_{l}(\mathbf{x})$ is the decision made for a given patch size $n_{l}=(2 l+1) 2,1 \leq l \leq L$ and $L$ is the number of sizes considered at each location. Let $\left(k_{D}(\mathbf{x})+1\right)$ be the number of positive decisions in $\mathcal{D}(\mathbf{x})$. The binary Bernouilli variables $D_{l}(\mathbf{x})$ are assumed to be not identically distributed and not really independent mainly because the patches with different sizes are nested. Nevertheless, according to the Chen-Stein method [11], $\sum_{l=1}^{L} D_{l}(\mathbf{x})$ is known to tend to a Poisson law $\operatorname{Po}\left(\lambda_{N}(\mathbf{x})\right)$ in distribution as $l \rightarrow \infty$ with $\lambda_{N}(\mathbf{x})=\sum_{l=1}^{L} \mathbf{P}_{H_{0}}\left(D_{l}(\mathbf{x})=1\right)$. To make a decision at location $\mathbf{x}$ from local decisions in the whole image, we assume first that $\lambda_{N}(\mathbf{x})$ defined as (Markov's inequality): $\lambda_{N}=\frac{e^{-N}}{|\Omega|} \sum_{l=1}^{L} \sum_{\mathbf{y} \in \Omega} e^{S_{N, l}(\mathbf{y})}$, is constant in the whole image. Finally, a change occurs at pixel if the probability of false alarm $\operatorname{PFA}(\mathbf{x})$ given by the Poisson tail with parameter $\lambda_{N}$ is lower than a desired level of significance $\alpha(\mathbf{x})$ :

$\operatorname{PFA}(\mathbf{x}) \triangleq \mathbf{P}_{H_{0}}\left(\sum_{l=1}^{L} D_{l}(\mathbf{x})>k_{D}(\mathbf{x})\right)=1-\sum_{k=0}^{k_{D}(\mathbf{x})}\left(\lambda_{N}\right)^{k} \frac{e^{-\lambda_{N}}}{k !}$.

By applying the Bonferroni strategy for multiple tests, we set $\alpha(\mathbf{x})=$ $\varepsilon /|\Omega|$ where $|\Omega|$ is the number of tested pixels and $\varepsilon$ is a user-defined expected number of false alarms over the whole image. We can refer to the a contrario framework [12] yielding the same control as Bonferroni while allowing one to set $\varepsilon \geq 1$ if needed. Setting $\varepsilon=1$ as we do in most experiments, means that about 1 pixel on average is falsely detected but the remaining detections are "meaningful' [12]. We define the final multiscale change detection $\operatorname{MCD}(u, v): \Omega \rightarrow$ $\{0,1\}$ as: $M C D(u, v)(\mathbf{x})=1$ if $\operatorname{PFA}(\mathbf{x}) \leq \varepsilon /|\Omega|$ and 0 otherwise. Our patch-based algorithm given in Fig. 2 is invariant to linear contrast changes applied to the image pair.

\subsection{Quantitative evaluation}

When used on experimental TIRF sequences, the detection is computed for each image pair. The chosen parameters were $L=4$ as maximum patch size, corresponding to $9 \times 9$ patches and $\varepsilon=1$ as indicated above. Finally, to account for the fact that only the events happening at the membrane are of interest, only those with a fluorescence level above a given threshold where examined. Because of the properties of TIRFM, the eventual detections with low fluorescence intensity correspond to events below the membrane and not relevant to recycling. Figure 3 shows a projection in maximum of a studied sequence with both manually and automatically detected appearances.

To quantitatively evaluate the accuracy of the detection, a ground truth was manually established for a couple of sequences, totalling a few hundreds of events. The detection accuracy was around $85 \%$ with a false positive rate below $10 \%$. Taking into account the difficulty of establishing a ground truth and the ambiguity of some events, such figures, which seemed robust across repeated experiments, are compatible with an automated analysis. Note that the use of micropatterned cell impose some stability to the results by reducing the variability of the experimental data.

\section{NORMALIZED REPRESENTATION FOR ANALYSIS}

Once detected, each appearance is supposed to correspond to one recycling event. Furthermore, once docked to the membrane the vesicle is immobile till its fusion with the plasma membrane. Thus the whole event is then characterized by the temporal variation of

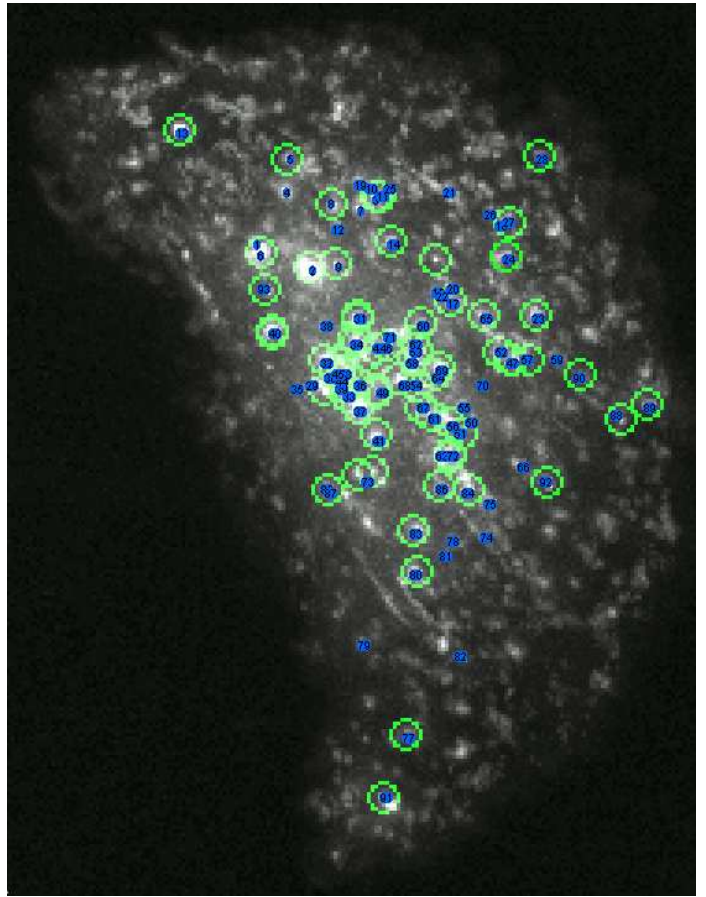

Fig. 3. Projection in maximum of a TfR-pHl sequence in TIRFM. In green the automatically detected appearances; in blue, the apperances labelled manually with numbers.

the fluorescence at that location. However, a robust characterisation of that temporal profile is complicated by the usual biological variability and various spatio-temporal perturbations; the computation of a normalized profile fit for systematic analysis and computation is needed.

Figure 4A shows such a temporal profile, with the detected appearance of a vesicle around frame \#100. The decrease can clearly be seen afterwards, up to frame \#200 or so. Depending on the particular studied protein, a biophysical model of that decrease, corresponding to diffusion in the membrane plane or other more complex mechanisms, might be available or hypothesised. We propose here to impose no strong constraint, to avoid being tied to a particular protein or model. It would also allow for comparison between proteins with different behaviours.

The only hypothesis we make is for the decrease to be convex. This corresponds to the property, in first approximation, of fluorescence of being additive with respect to concentration. Thus any perturbation can be detected as concave addition to a convex decreasing trend; they can be disregarded by focusing on an approximation of the curve from below. The first step of the processing is the computation of the ending time of the event. Following the hypothesis of a convex decrease, that ending time is looked for as corresponding to the first 'significant' local minimum after the detection, thus to the first 'big' concavity. To that end, the lower $\alpha$-scale space [13] is used, a geometrical multi-scale analysis framework that use local convexity to build scaled version from the signal itself to its convex hull. Figure 4B shows the result for 3 values of $\alpha$, with the corresponding computed ending times numbered 1,2 and 3 . The chosen value of $\alpha$ is calibrated in time with respect to the studied process, and thus is stable across experiments.

Still following the convex hypothesis, the normalised version of the recycling event is computed as the lower convex hull of the sig- 


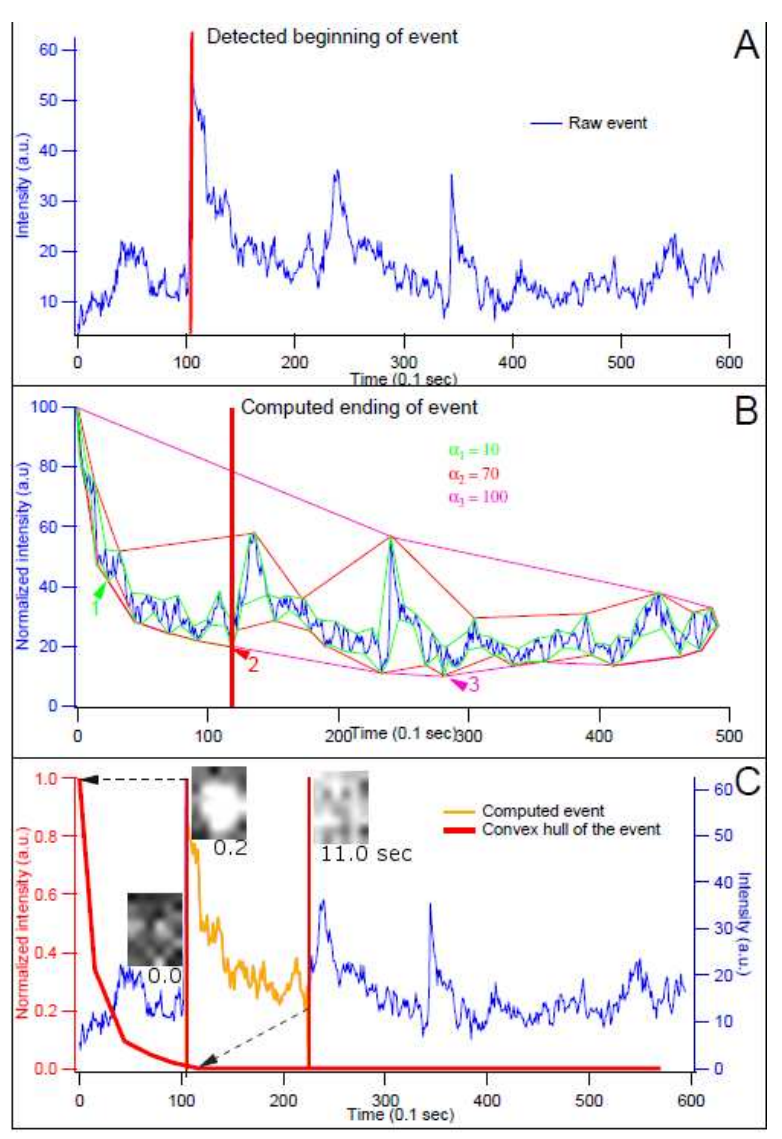

Fig. 4. A. The raw signal, B. $\alpha$-scale space to compute the end of the event, C. in red the normalized decrease fit for analysis.

nal between the beginning and the end of the event. The resulting profile is shown figure $4 \mathrm{C}$ in red, scaled between 0 and 1 . Figure $5 \mathrm{~A}$ shows all the detected recycling events for one of the studied 600 frames sequence of TfR in TIRFM. Thanks to the proposed normalisation procedure, further studies at the cell wide level are then enabled, including: i) computation of the half life of the decreases (cf Fig. 5B), ii) comparison between individual events to distinguish between biophysical mechanisms, iii)analysis of perturbations of the biological model. Statistical machine learning algorithms with geometrical distances between curves will be used in future work.

\section{CONCLUSION}

TIRFM, by focusing on the cellular membrane, allows for the precise study of endocytosis/recycling and exocytosis pathways. This work based on the recycling of transmembrane protein, proposes a framework for the detection and representation of individual recycling events, including a novel statistical detection algorithm. TfR was used as a case study, and the evaluation of the detections showed results almost fully compatible with a manual detection approach.

Current work includes the validation of the normalized representation using $\alpha$-scale space, which could not be included here. Among biological questions, this framework enables us to study the comparison of the recycling of TfR and another less studied transmenbrane protein, the Langerine. Future plans include the use of dual-color TIRFM to compare the spatiotemporal dynamics of the recycling of a transmembrane protein along with others proteins that constitute the complex machinery of the cell involved in the molecular mechanisms of membrane recycling.

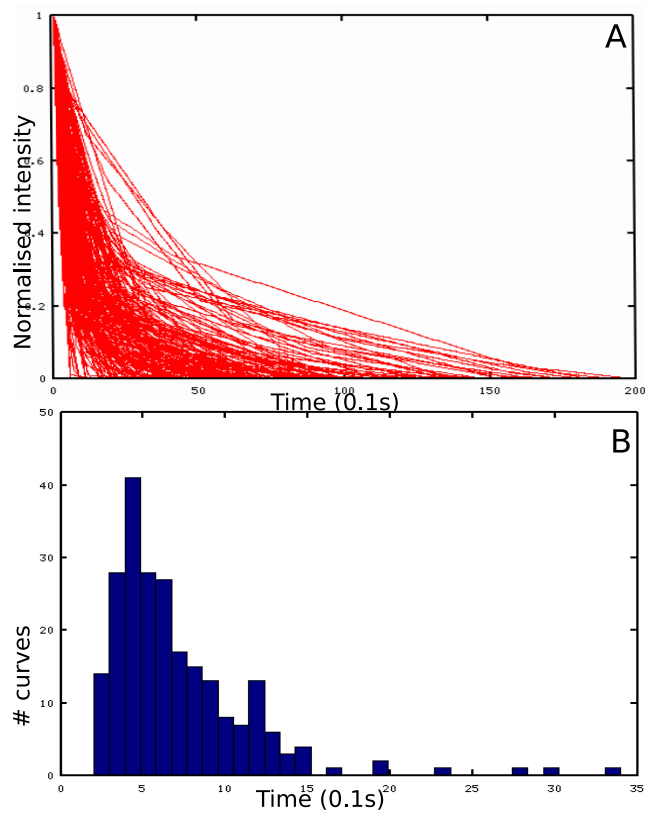

Fig. 5. Normalised decreases for one TfR-pHl TIRFM sequence; B. corresponding histogram of half-life.

\section{REFERENCES}

[1] J.D. Bleil and M.S. Bretscher, "Transferrin receptor and its recycling in hela cells," EMBO J., vol. 1, no. 3, pp. 351-355, 1982.

[2] B.D. Grant and J.G. Donaldson, "Pathways and mechanisms of endocytic recycling," Nat. Rev. Mol. Cell. Biol., vol. 10, pp. 597-608, 2009.

[3] E.W. Mllner, B. Neupert, and L.C. Khn, "A specific mrna binding factor regulates the iron-dependent stability of cytoplasmic transferrin receptor mrna," Cell, vol. 58, no. 2, pp. 376-382, 1989.

[4] M.E. Daz, G. Ayala, T. Len, R Zoncu, and D. Toomre, "Analyzing protein-protein spatial-temporal dependencies from image sequences using fuzzy temporal random sets," J. Comput. Biol., vol. 15, no. 9, pp. 1221-1236, 2008.

[5] R. Sebastian, M.E. Diaz, G. Ayala, K. Letinic, J. Moncho-Bogani, and D. Toomre, "Spatio-temporal analysis of constitutive exocytosis in epithelial cells," IEEE/ACM Trans. Comput. Biol. Bioinform., vol. 3, no. 1, pp. 17-32, 2006.

[6] C.J. Merrifield, D. Perrais, and D. Zenisek, "Coupling between clathrin-coated-pit invagination, cortactin recruitment, and membrane scission observed in live cells.," Cell, vol. 121, pp. 593-606, 2005.

[7] R. Niu, P. Varshney, M. Moore, and D. Klamer, "Decision fusion in a wireless sensor network with a large number of sensors," in Proc. FUSION'04, Stockholm, Sweden, 2004.

[8] S. Ince and J. Konrad, "Geometry-based estimation of occlusions form video frame pairs," in Proc. ICASSP'05, Philadelphia, USA, 2005.

[9] P.M. Jodoin, M. Minette, and C. Rosenberger, "Segmentation framework based on label field fusion," IEEE T. Signal Processing, vol. 16, pp. 2535-2550, 2007.

[10] N. Katenka, E. Levina, and G. Michailidis, "Local vote decision fusion for target detection in wireless sensor netwroks.," IEEE T. Signal Processing, vol. 56, pp. 329-338, 2008.

[11] R. Arratia, L. Goldstein, and L. Gordon, "Two moments suffice for poisson approximations: the chen-stein method," Annals of Probability, vol. 17, pp. 9-25, 1989.

[12] A. Desolneux, L. Moisan, and J.M. Morel, "Meaningful alignments," Int. J. Comp. Vis., vol. 40, pp. 7-23, 2000.

[13] A. Chessel, B. Cinquin, S. Bardin, J. Salamero, and Ch. Kervrann, "Computational geometry-based scale-space and modal image decomposition," in Proc. SSVM'09, Voss, Norway, June 2009. 